\section{Reply: High Sensitivity \\ of Indocyanine Green \\ Fluorescence Imaging in Detection of Sentinel Node}

\section{TO THE EDITOR,}

The argument by Takahashi et al. has been discussed in our article. ${ }^{1}$ The following is our brief response.

Kitai et al. noted that the sensitivity of fluorescence spectroscopy is greater than that of absorption spectroscopy. ${ }^{2}$ They also reported that it was possible to detect fluorescence with an indocyanine green (ICG) solution embedded $10 \mathrm{~mm}$ deep in a material that has optical properties like that of human tissue. In comparison, infrared rays can penetrate fatty tissues only to a depth of $3 \mathrm{~mm}$. The difference could be critical in sentinel node detection. In this context, Ishikawa et al. reported an obese patient with a false-negative sentinel node when the infrared ray electronic endoscopy system was used. ${ }^{3}$

We reported that the ICG fluorescence imaging system was sensitive in both intraoperative ICG injection and ICG injection 1 day before surgery. We have argued that, similar to the radio-guided method, one advantage of preoperative tracer injection is that it eliminates the timeconsuming intraoperative endoscopy, but has the disadvantage of loss of real-time tracing. ${ }^{1}$

An astral lamp, but not an ordinary light lamp, influences the ICG fluorescence imaging system. Therefore, surgery can be continued under an ordinary light lamp, which should not interfere with the ICG fluorescence imaging system. Furthermore, the light emitted by the laparoscope into the peritoneal cavity does not influence the ICG fluorescence imaging system. Laparoscopic exploration or surgery can be conducted under such an environment.

The laparoscopic system described in our report is not available commercially. ${ }^{1}$ We have completed a preliminary study with a newly developed prototype system for laparoscopic surgery and are preparing a new report on its use in laparoscopic surgery.

\section{Isao Miyashiro, MD}

Department of Surgery, Osaka Medical Center for Cancer and Cardiovascular Diseases, 1-3-3 Nakamichi, Higashinari-ku, Osaka 537-8511, Japan

e-mail: miyashir@biken.osaka-u.ac.jp

Published Online: 21 March 2009

(C) Society of Surgical Oncology 2009

\section{REFERENCES}

1. Miyashiro I, Miyoshi N, Hiratsuka M, et al. Detection of sentinel node in gastric cancer surgery by indocyanine green fluorescence imaging: comparison with infrared imaging. Ann Surg Oncol. 2008;15:1640-3.

2. Kitai $\mathrm{T}$, Inomoto $\mathrm{T}$, Miwa $\mathrm{M}$, et al. Fluorescence navigation with indocyanine green for detecting sentinel lymph nodes in breast cancer. Breast Cancer. 2005;12:211-5.

3. Ishikawa K, Yasuda K, Shiromizu A, et al. Laparoscopic sentinel node navigation achieved by infrared ray electronic endoscopy system in patients with gastric cancer. Surg Endosc. 2007;21: $1131-4$. 